\title{
Modelling the disturbance caused by a dc-electrified railway to geomagnetic measurements
}

\author{
Risto Pirjola ${ }^{1,2}$, Larry Newitt $^{1}$, David Boteler ${ }^{1}$, Larisa Trichtchenko ${ }^{1}$, Peter Fernberg ${ }^{1}$, Lorne McKee ${ }^{1}$, \\ Donald Danskin ${ }^{1}$, and Gerrit Jansen van Beek ${ }^{1}$ \\ ${ }^{1}$ Natural Resources Canada, Geomagnetic Laboratory, 7 Observatory Crescent, Ottawa, Ontario, K1A 0Y3, Canada \\ ${ }^{2}$ Finnish Meteorological Institute, Space Research Unit, P.O. Box 503, FIN-00101 Helsinki, Finland
}

(Received October 12, 2006; Revised May 29, 2007; Accepted May 29, 2007; Online published August 31, 2007)

\begin{abstract}
Magnetic fields created by a dc-electrified railway are a nuisance to the operation of a geomagnetic observatory and also disturb other electromagnetic studies. Theoretical formulas that enable quantitative estimates of the magnetic effect of a dc railway including leakage currents in the ground are presented in this paper. They are illustrated by numerical examples. The validity of the theoretical model was verified by measurements carried out in the vicinity of a nearly north-south railway in Calgary, Canada. The earth structure in that area is approximately layered, which is an assumption included in the theoretical model. The agreement between the measured magnetic fields due to trains and the theoretical values is good. Numerical computations indicate that magnetic fields larger than the maximum allowable noise level (assumed to be about $10 \mathrm{pT}$ ) at today's magnetic observatories may extend to distances of tens of kilometres from a railway. We have prepared computer programs based on the theoretical formulas in the MatLab, Octave, FORTRAN and IDL languages, in which the locations (i.e. the latitudes and the longitudes) of the point of observation, of the feeding substations and of the trains, together with the feeding and leakage currents and the heights of the feeding lines, can be given as inputs.
\end{abstract}

Key words: Magnetic observatory, dc train, leakage current, dc magnetic field.

\section{Introduction}

Electric currents create magnetic fields that may seriously disturb geomagnetic observatory recordings and other electromagnetic studies even at distances of tens of kilometres from the source. Particularly harmful in this respect are dc-electrified railways (Yanagihara, 1977; Tokumoto and Tsunomura, 1984; Lowes, 1987; Iliceto and Santarato, 1999; Egbert et al., 2000; Georgescu et al., 2002; Pádua et $a l ., 2002)$. In principle, the current used for feeding trains flows in a closed circuit consisting of an overhead feeding line and the return path along the rails. Since the wire and the return path are close to each other and carry the same current in opposite directions their magnetic effects tend to cancel each other, and a significant magnetic field can only be observed near the railway. In practice, however, the rails are not insulated from the ground. Iliceto and Santarato (1999) even point out that, for safety reasons, rails are carefully earthed at points about $100 \mathrm{~m}$ apart. Consequently, a large amount of leakage current flows into the earth, and the cancellation mentioned does not occur and additionally the leakage current, which may extend to a long distance in the ground, also produces a magnetic field. Besides the magnetic effect, an electrified railway is naturally also accompanied by an electric field (Kovalevskiy et al., 1961; Iliceto and Santarato, 1999; Georgescu et al., 2002; Pádua et al., 2002; Tanbo et al., 2003).

Copyright (c) The Society of Geomagnetism and Earth, Planetary and Space Sciences (SGEPSS); The Seismological Society of Japan; The Volcanological Society of Japan; The Geodetic Society of Japan; The Japanese Society for Planetary Sciences; TERRAPUB.
The theoretical model introduced by Georgescu et al. (2002) for calculating the magnetic field created by a dcelectrified train with a substation and a feeding line is adopted in Section 2. However, the presentation and the derivation of the pertinent equations are independent in this paper, so actually a kind of a confirmation of the work by Georgescu et al. (2002) is obtained. Computer codes based on the formulas thus enabling the estimation of the magnetic disturbances by a railway in different configurations have been prepared in the MatLab, Octave, FORTRAN and IDL languages. Numerical examples are presented in Section 3 . To verify the validity of the theoretical model, measurements of the magnetic field at different distances from a railway were carried out in Calgary, Canada. They and their good agreement with calculated results are discussed in Section 4.

\section{Theoretical Model}

We consider a rectangular train-feeding substation loop of length $L$ and height $h$ shown in Fig. 1. As pointed out in Section 1, the model is the same as that presented by Georgescu et al. (2002) but the calculations of the magnetic field are performed independently now. The current injected by the substation via the feeding line into the train is $J_{1}$. Let us use a right-handed Cartesian $x y z$ coordinate system in which the $x$ axis is parallel to the loop with the substation and the train located at $x=0$ and $x=L$, respectively, and the $z$ axis points downwards. The bottom of the loop lies at the $x y$ plane (= the earth's surface). It is thus assumed in the model that the feeding line and the rails between the substation and the train are straight. 


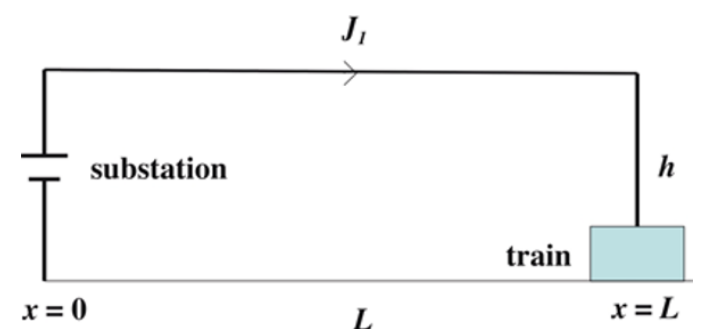

Fig. 1. Rectangular train-substation loop including the feeding line of a length $L$ and height $h$. The feeding current is $J_{1}$. The $x$ axis is assumed to be parallel to the loop so that the feeder and the train are located at $x=0$ and $x=L$, respectively.

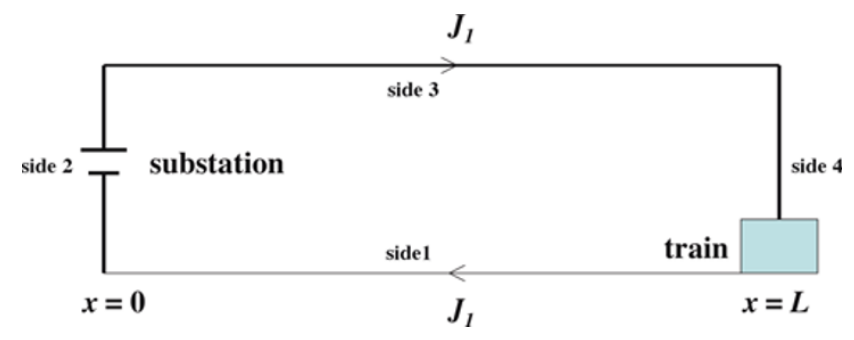

Fig. 2. Rectangular "full" train-substation loop (width $=L$, height $=h$ ) with the feeding current $J_{1}$. The sides of the loop are numbered: $1=$ rails, $2=$ substation, $3=$ feeding line, $4=$ train.

In the ideal case, the feeding current should return from the train to the substation along the rails, i.e. from $x=L$ to $x=0$ at $z=0$, so that a "full" rectangular $J_{1}$ loop depicted in Fig. 2 is considered. In practice, however, the rails are not insulated from the ground, and so there is a significant current leakage from the rails into the ground. Similarly to Georgescu et al. (2002), we assume that the leakage current returns to the system at the substation, which is justified by the low grounding resistance of the station.

The earth's conductivity is assumed to be uniform or layered, so that the leakage currents flow radially symmetrically from the point of injection to all directions in the ground. This means that when considering the magnetic field at the earth's surface we may replace the symmetrically-flowing current by a single semi-infinite downward vertical line current (Yanagihara, 1977; Tokumoto and Tsunomura, 1984). Note that Yanagihara explicitly speaks about a homogeneous earth, which is too strict an assumption since inhomogeneities in the vertical direction are acceptable. Replacing a radial distribution by a single vertical current is actually a modification of the wellknown "Fukushima theorem" widely used when studying magnetic fields on the ground due to magnetosphericionospheric currents (Fukushima, 1976). Its validity can be easily proved by applying Ampére's law, which states that, when neglecting the displacement currents, the line integral of the magnetic field around any closed loop equals the total current through the loop multiplied by the permeability. The equivalence between a radially symmetric current distribution and a semi-infinite vertical current also holds true for a time-dependent situation provided the displacement currents can still be neglected. This corresponds to the real situation in which the feeding current varies following the

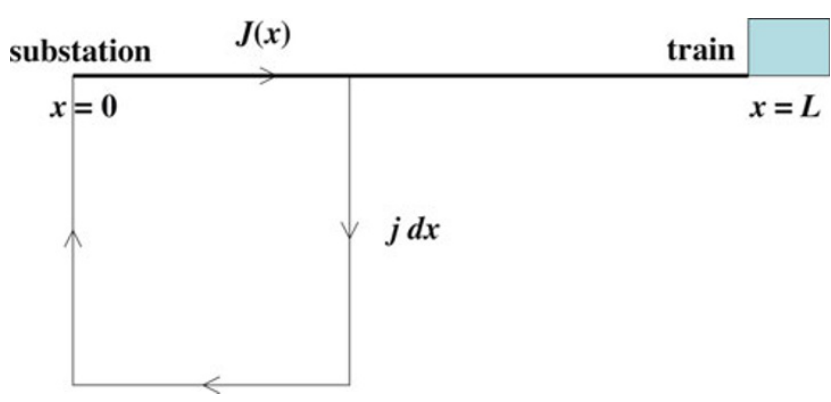

Fig. 3. Rectangular "leakage" loop with the current $j d x$. The amount of the "leakage" current at the point $x$, which decreases the "full"-loop current ( $J_{1}$ in Fig. 1$)$, is $J(x)=j(L-x)$. The bottom horizontal part of the loop lies at infinity.

acceleration and deceleration activities of the train.

We assume that the leakage is constant $(=j d x)$ along the rails, i.e. the leakage current density per unit length $(=j)$ is the same at each infinitesimal section $[x, x+$ $d x$ ] between $x=L$ and $x=0$. Thus, in addition to the ideal "full" loop depicted in Fig. 2, we also have a set of "leakage" loops (Fig. 3). Consequently, the current that actually flows along the rails at a point $x$ towards the substation is

$$
J_{\text {rail }}(x)=J_{1}-\int_{x}^{L} j d x=J_{1}-j(L-x) .
$$

The latter term $j(L-x)$ represents the decrease of $J_{1}$ due to the leakage and can be described as an opposite "leakage" current as indicated by $J(x)$ in Fig. 3. The total "leakage" current between $x=L$ and $x=0$, denoted by $J_{0}$, equals $J(0)=j L$, and so $j=J_{0} / L$.

To calculate the magnetic field at a point $(x, y)$ at the earth's surface $(z=0)$, we thus have to add the contributions from the "leakage" loops $j d x=J_{0} d x / L$ to the magnetic field produced by the "full" loop. The magnetic field element $\mathbf{d B}$ caused by any (vector) current element $I \mathbf{~ d s}$ is obtained from the Biot-Savart law:

$$
\mathbf{d B}=\frac{\mu_{o} I}{4 \pi} \frac{\mathbf{d s} \times \mathbf{r}}{r^{3}}
$$

where $\mu_{0}$ is the (vacuum) permeability and $\mathbf{r}$ is the vector from the current element to the point of observation $(r=$ $|\mathbf{r}|)$. All currents included in the present problem and shown in Figs. 2 and 3 are straight and have a finite or semi-infinite length. Application of Eq. (2) to a straight current line $I$ easily leads to the magnetic field expression

$$
\mathbf{B}=\frac{\mu_{0} I}{4 \pi R}\left(\cos \theta_{1}-\cos \theta_{2}\right) \mathbf{e}_{\varphi}
$$

where $R$ is the perpendicular distance of the point of observation from the line determined by the current, $\mathbf{e}_{\varphi}$ is a unit vector around the line in a cylindrical coordinate system, and $\theta_{1}$ and $\theta_{2}$ are the angles between the line and the vectors from the ends of the current to the point of observation. (For an infinitely long line current, $\theta_{1}$ and $\theta_{2}$ are $0^{\circ}$ and $180^{\circ}$, so that Eq. (3) reduces to the well-known form $\mathbf{B}=\mu_{0} I / 2 \pi R \mathbf{e}_{\varphi}$.)

Equation (3) and somewhat tedious geometrical considerations enable the calculation of the magnetic field at a 
point $(x, y, z=0)$ due to the different currents shown in Figs. 2 and 3. The contribution from the "full" loop is

$$
\begin{aligned}
B_{x}^{F}= & B_{2} \frac{y}{\sqrt{y^{2}+x^{2}}}-B_{4} \frac{y}{\sqrt{y^{2}+(L-x)^{2}}} \\
B_{y}^{F}= & -B_{2} \frac{x}{\sqrt{y^{2}+x^{2}}}-B_{3} \frac{h}{\sqrt{y^{2}+h^{2}}} \\
& -B_{4} \frac{L-x}{\sqrt{y^{2}+(L-x)^{2}}} \\
B_{z}^{F}= & -B_{1}+B_{3} \frac{y}{\sqrt{y^{2}+h^{2}}}
\end{aligned}
$$

where the $B_{k}(k=1,2,3,4)$ quantities are the fields created by the four sides of the "full" loop. As shown in Fig. 2, the side numbers are 1 for the bottom (rails), 2 for the lefthand (substation), 3 for the top (feeding line) and 4 for the right-hand side (train).The expressions of the $B_{k}$ quantities are

$$
\begin{aligned}
B_{1}= & \frac{\mu_{0} J_{1}}{4 \pi y}\left(\frac{L-x}{\sqrt{y^{2}+(L-x)^{2}}}+\frac{x}{\sqrt{y^{2}+x^{2}}}\right) \\
B_{2}= & \frac{\mu_{0} J_{1}}{4 \pi \sqrt{y^{2}+x^{2}}} \frac{h}{\sqrt{y^{2}+x^{2}+h^{2}}} \\
B_{3}= & \frac{\mu_{0} J_{1}}{4 \pi \sqrt{y^{2}+h^{2}}}\left(\frac{x}{\sqrt{y^{2}+x^{2}+h^{2}}}\right. \\
& \left.+\frac{L-x}{\sqrt{y^{2}+(L-x)^{2}+h^{2}}}\right) \\
B_{4}= & \frac{\mu_{0} J_{1}}{4 \pi \sqrt{y^{2}+(L-x)^{2}}} \frac{h}{\sqrt{y^{2}+(L-x)^{2}+h^{2}}} .
\end{aligned}
$$

We now apply Eq. (3) to the sides of a single elementary "leakage" loop carrying the current $j d x^{\prime}$, which has the upward and downward vertical parts at $x=0$ and at $x=x^{\prime}$ (see Fig. 3). The vertical sides of the loop only contribute to the magnetic $x$ and $y$ components at the point of observation $(x, y, z=0)$ whereas the upper horizontal part only creates a $z$-directed field. The lower horizontal part lying at an infinite distance does not contribute to the field at the earth's surface. Consequently and after some geometrical considerations, we obtain the following expressions for the magnetic field due to the elementary "leakage" loop $j d x^{\prime}$ :

$$
\begin{aligned}
& d B_{x}=-\frac{\mu_{0} j d x^{\prime}}{4 \pi}\left(\frac{y}{y^{2}+\left(x^{\prime}-x\right)^{2}}-\frac{y}{y^{2}+x^{2}}\right) \\
& d B_{y}=-\frac{\mu_{0} j d x^{\prime}}{4 \pi}\left(\frac{x^{\prime}-x}{y^{2}+\left(x^{\prime}-x\right)^{2}}+\frac{x}{y^{2}+x^{2}}\right) \\
& d B_{z}=\frac{\mu_{0} j d x^{\prime}}{4 \pi y}\left(\frac{x^{\prime}-x}{\sqrt{y^{2}+\left(x^{\prime}-x\right)^{2}}}+\frac{x}{\sqrt{y^{2}+x^{2}}}\right) .
\end{aligned}
$$

To obtain the total magnetic field, the contributions from all "leakage" loops $j d x^{\prime}$ have to be summed. In other words, Eqs. (11)-(13) are integrated over $x^{\prime}$ from $x^{\prime}=0$ to $x^{\prime}=L$. The integral functions of all terms are elementary functions, and the final result of the magnetic contribution from the "leakage" loops is

$$
B_{x}^{L}=-\frac{\mu_{0} J_{0}}{4 \pi L}\left(\overline{\operatorname{arc}} \tan \left(\frac{L-x}{y}\right)\right.
$$

$$
\begin{gathered}
\left.+\overline{\operatorname{arc} t a n}\left(\frac{x}{y}\right)-\frac{y L}{y^{2}+x^{2}}\right) \\
B_{y}^{L}=-\frac{\mu_{0} J_{0}}{4 \pi L}\left(\ln \left(\frac{\sqrt{y^{2}+(L-x)^{2}}}{\sqrt{y^{2}+x^{2}}}\right)+\frac{x L}{y^{2}+x^{2}}\right) \\
B_{z}^{L}=\frac{\mu_{0} J_{0}}{4 \pi y L}\left(\frac{x L}{\sqrt{y^{2}+x^{2}}}\right. \\
\left.+\sqrt{y^{2}+(L-x)^{2}}-\sqrt{y^{2}+x^{2}}\right) .
\end{gathered}
$$

(Note that $j=J_{0} / L$.) Finally, the contributions from the "full" loop and from the "leakage" loops have to be added together:

$$
B_{u}^{\text {TOTAL }}=B_{u}^{F}+B_{u}^{L}
$$

where the subscript $u$ refers to $x, y$ or $z$.

Note that the $x y$ coordinate system at the earth's surface was defined by the train-feeding substation system, so when considering the magnetic field produced by several trains simultaneously we have many different $x y$ systems. Naturally, the final result, i.e. the magnetic field components at the point of observation, should be obtained in a fixed coordinate system, and the most convenient way in this respect is to give the geographic north and east components and the downward vertical $(=z)$ component of the magnetic field.

We have prepared computer programs in the MatLab, Octave, FORTRAN and IDL languages, which enable the calculation of the magnetic field at an arbitrary point of observation due to several train-feeding substation pairs. Besides the information about the feeding current systems, i.e. the parameters $L, h, J_{1}$ and $J_{0}$, the program input includes a specification of the geographic direction of each substationtrain pair and of the location of the point of observation in the individual $x y$ coordinate systems associated with each substation-train pair. To make the programs easier for a user to apply, modified versions have also been constructed in which the latitudes and longitudes of the point of observation, of the substations and of the trains (together with $h, J_{1}$ and $J_{0}$ ) are required as the input and the programs compute the lengths $L$, the directions and the $x$ and $y$ coordinates.

\section{Numerical Results}

The equations presented in the previous section and the computer programs now enable calculations of the magnetic fields created by different train-feeding substation configurations at any point of observation. Most of the computations made so far are based on the MatLab versions. We now present three numerical examples.

Let us first consider a straight north-south railway of 10 $\mathrm{km}$ in length. Although it well simulates the situation in Calgary, Canada, in which measured data discussed in Section 4 were collected, the particular geographical location is of no importance in this example. We assume that the railway is occupied (possibly somewhat unrealistically) by altogether twenty trains such that there are two trains in opposite directions at $1 \mathrm{~km}$ intervals with no trains at the southern end. It is further assumed that each train, independently of its moving direction, is fed from the south by a current of $1000 \mathrm{~A}\left(=J_{1}\right)$ with a feeding wire of $1 \mathrm{~km}(=L)$ in 


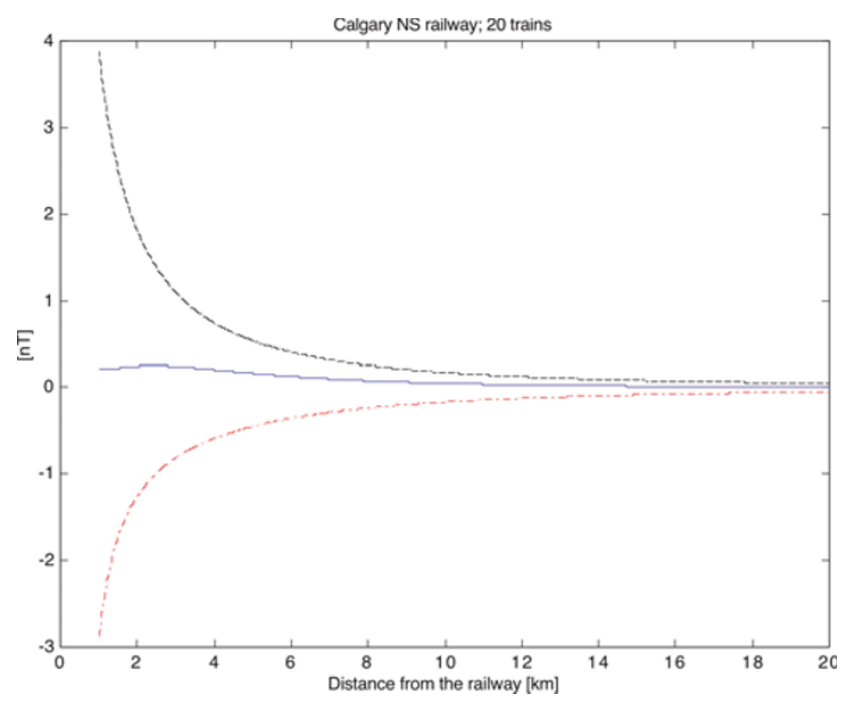

Fig. 4. Magnetic field created by a $10-\mathrm{km}$ straight north-south dc railway (simulating the situation in Calgary, Canada, though the particular geographical location is of no importance here). The field components (north $=$ solid blue, east=dot-dashed red, downward vertical=dashed black) are shown as functions of the eastward perpendicular distance from the railway along a line located $3 \mathrm{~km}$ north of the southern end of the railway. It is assumed that there are twenty trains, one in both directions at $1 \mathrm{~km}$ intervals (no trains at the southern end). It is further assumed that each train, independently of its direction, is fed from the south by a current of $1000 \mathrm{~A}\left(=J_{1}\right)$ with a feeding wire of $1 \mathrm{~km}(=L)$ in length and $5 \mathrm{~m}(=h)$ in height. The total leakage current is $20 \mathrm{~A}$ $\left(=J_{0}\right)$ for all trains.

length and $5 \mathrm{~m}(=h)$ in height and that the total leakage current is $20 \mathrm{~A}\left(=J_{0}\right)$. (Note that the value of $1000 \mathrm{~A}$ for the feeding current $J_{1}$ may be considered a lower limit since Georgescu et al. (2002) mention a maximum magnitude of $3500 \mathrm{~A}$, and Iliceto and Santarato (1999) also indicate values of more than 1000 A.) Figure 4 depicts the magnetic field components (north=solid blue, east=dot-dashed red, downward vertical=dashed black) as functions of the eastward perpendicular distance from the railway along a line located $3 \mathrm{~km}$ north of the southern end of the railway. It is seen that the field is very large near the railway but drops off rapidly with the distance. Due to the geometry, the north component is clearly smaller than the two other components in this case.

The nominal total leakage current can be assumed to equal $20 \mathrm{~A}$ as is the case in Fig. 4. Georgescu et al. (2002), however, point out that sometimes more than $50 \%$ of the feeding current may leak into the ground. Therefore, in the second example, we discuss the magnetic field components at a given site as functions of the total leakage current. A railway section of $2.5 \mathrm{~km}$ with two trains travelling in opposite directions at the end of the railway and fed by a substation at the other end is considered so that $L=2.5 \mathrm{~km}$. These assumptions imply that both train-feeding substation pairs define the same $x y$ coordinate system. The point of observation is set at $x=10.5 \mathrm{~km}$ and $y=8 \mathrm{~km}$. This configuration refers to the calculation of the magnetic disturbance that would occur at the Ottawa Magnetic Observatory, Canada, if a dc railway section would be constructed in downtown Ottawa according to one of the plans in the future but in this paper the calculation is just regarded as an

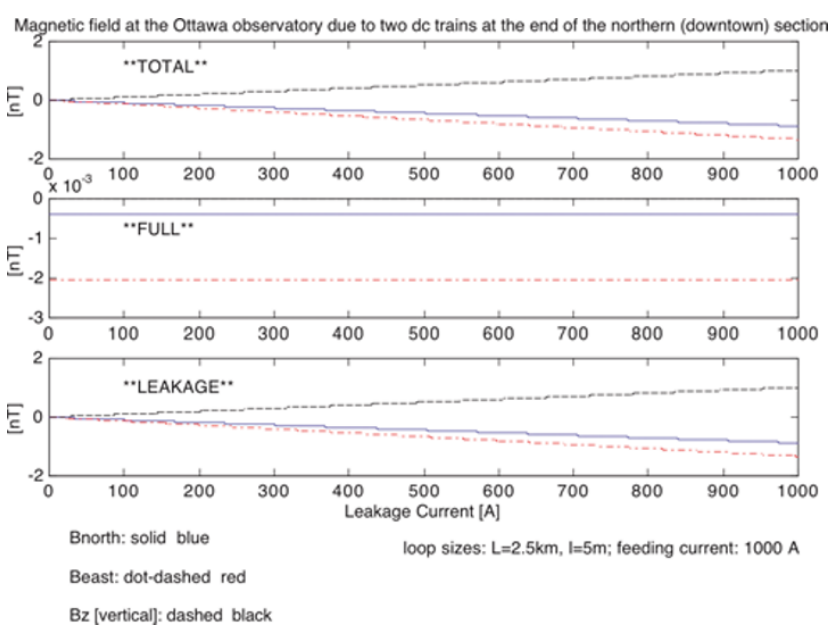

Fig. 5. Magnetic field created by a $2.5-\mathrm{km}$ dc railway It is assumed that there are two trains at the end of the railway travelling in opposite directions and powered by a substation at the other end of the railway. So the lengths of the feeding wires are $2.5 \mathrm{~km}(=L)$, and the heights of the feeding wires and the feeding currents are assumed to be $5 \mathrm{~m}$ $(=h)$ and $1000 \mathrm{~A}\left(=J_{1}\right)$. The magnetic field is calculated at a point having coordinates $x=10.5 \mathrm{~km}$ and $y=8 \mathrm{~km}$ with respect to the train-feeding substation systems (see the text and Fig. 1 for the definition of the coordinates). Referring to Fig. 6(a), the situation corresponds to the magnetic field at the point of observation $(\mathrm{P}-\mathrm{O}-\mathrm{O})$ due to two $\mathrm{T} 1+\mathrm{F} 1$ train-feeding substation pairs (but with $L=2.5$ $\mathrm{km}$ now). The field components (north $=$ solid blue, east $=$ dot-dashed red, downward vertical=dashed black) are presented as functions of the total leakage current $\left(=J_{0}\right)$, which is the same for both trains. The figure also shows the contributions from the "full" and "leakage" loops separately. (The parameter values used in this figure correspond to the magnetic field at the Ottawa Magnetic Observatory, Canada, due to a planned dc railway section though the particular geographical area is of no importance here.)

example. The feeding currents $J_{1}$ and the heights $h$ of the feeding wires are set to $1000 \mathrm{~A}$ and $5 \mathrm{~m}$. Figure 5 shows the field components (north=solid blue, east=dot-dashed red, downward vertical=dashed black) as functions of the total leakage current $\left(=J_{0}\right)$, which is the same for both trains, and the contributions from the "full" $\left(B_{\text {north,east }, z}^{F}\right)$ and "leakage" ( $\left.B_{\text {north,east, } z}^{L}\right)$ loops are also depicted separately. We can see that the total field is dominated by the "leakage" part. Note that $B_{z}^{F}$ even remains so small that it is invisible in the figure. The "full" contributions are, of course, constant all over the figure and have the following values: $B_{\text {north }}^{F}=-4.1210^{-4} \mathrm{nT}, B_{\text {east }}^{F}=-2.0510^{-3} \mathrm{nT}$, $B_{z}^{F}=-5.6310^{-7} \mathrm{nT}$. For the nominal leakage current $J_{0}=20 \mathrm{~A}$, the total and "leakage" fields have the following values: $B_{\text {north }}^{\text {TOTAL }}=-0.0179 \mathrm{nT}, B_{\text {east }}^{\text {TOTAL }}=-0.0286$ $\mathrm{nT}, B_{z}^{\mathrm{TOTAL}}=0.0204 \mathrm{nT}, B_{\text {north }}^{L}=-0.0175 \mathrm{nT}, B_{\text {east }}^{L}=$ $-0.0265 \mathrm{nT}, B_{z}^{L}=0.0204 \mathrm{nT}$.

In the third example, we consider the configuration shown in Fig. 6(a), in which an approximately north-south railway consists of four straight sections. The configuration corresponds to a dc railway alternative included in the plans for Ottawa with the point of observation $(\mathrm{P}-\mathrm{O}-\mathrm{O})$ being the Ottawa Magnetic Observatory although the particular geographical area is of no importance in this example. Figure 6(b) depicts the absolute value of the magnetic field as a function of the distance (along the Distance Axis in Fig. 6(a)) from the railway due to one train-feeding sub- 

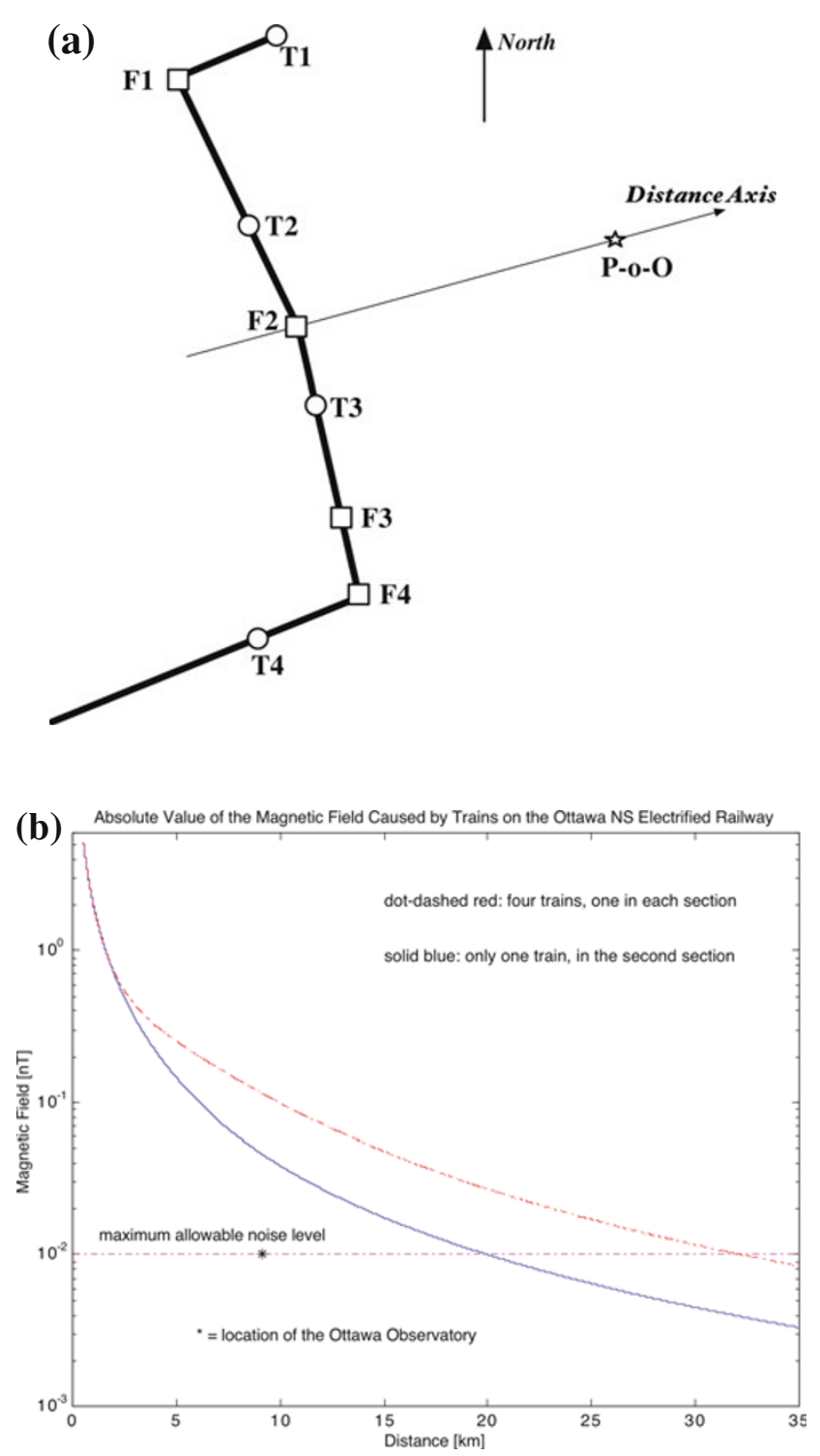

Fig. 6. (a) Approximately north-south-oriented dc railway configuration used to calculate the magnetic field produced at the Distance Axis. A particular point of observation is denoted by "P-o-O" and a star. The railway consists of four linear sections with one train-feeding substation pair $(\mathrm{Tk}+\mathrm{Fk}, k=1,2,3,4)$ in each of them. The distances between the train and the substation equal $3 \mathrm{~km}(=L)$. The distance between $\mathrm{F} 2$ and $\mathrm{P}-\mathrm{o}-\mathrm{O}$ is about $9.1 \mathrm{~km}$. (The configuration corresponds to a planned dc railway in Ottawa, Canada, with P-o-O being the Ottawa Magnetic Observatory though the particular geographical area is of no importance in this paper.) (b) Magnetic field created by an approximately north-south de railway shown in Fig. 6(a) and consisting of four straight sections. It is assumed that there is one train-feeding substation pair (T2+F2 in Fig. 6(a)) located in the second section (solid blue curve) or four train-feeding substation pairs $(\mathrm{T} 1+\mathrm{F} 1, \mathrm{~T} 2+\mathrm{F} 2, \mathrm{~T} 3+\mathrm{F} 3$ and $\mathrm{T} 4+\mathrm{F} 4$ in Fig. 6(a)) located one in each section (dot-dashed red curve). In each case, the distance between the feeder and the train equals $3 \mathrm{~km}$ $(=L)$, and the heights of the feeding wires and the feeding currents are assumed to be $5 \mathrm{~m}(=h)$ and $1000 \mathrm{~A}\left(=J_{1}\right)$. The total leakage currents are $20 \mathrm{~A}\left(=J_{0}\right)$. Although the configuration is a simulation of a planned situation in Ottawa, Canada, the particular geographical location is unimportant here. The absolute value of the magnetic field is presented as a function of the distance from the railway (Distance Axis in Fig. 6(a)). The asterisk denotes the location of the point of observation (P-o-O in Fig. 6(a) simulating the Ottawa Magnetic Observatory). The straight line at $10^{-2} \mathrm{nT}$ shows the highest acceptable disturbance level at a high-standard observatory today. station pair (T2 + F2 in Fig. 6(a)) in the second section (solid blue curve) or due to four pairs $(\mathrm{T} 1+\mathrm{F} 1, \mathrm{~T} 2+\mathrm{F} 2$, $\mathrm{T} 3+$ F3 and T4 + F4 in Fig. 6(a)) one located in each section (dot-dashed red curve). For all pairs, the distance between the train and the substation is $3 \mathrm{~km}(=L)$, and the heights of the wires and the feeding currents are assumed to be $5 \mathrm{~m}(=h)$ and $1000 \mathrm{~A}\left(=J_{1}\right)$. The total leakage currents are $20 \mathrm{~A}\left(=J_{0}\right)$. As the origin of the distance shown in Fig. 6(b) is at the feeding substation (F2), the influence of $\mathrm{T} 2+\mathrm{F} 2$ completely dominates near the railway, and only further away the dot-dashed curve is clearly above the solid curve. We can see that both for one train and for four trains the magnetic field at the point of observation $(\mathrm{P}-$ o-O in Fig. 6(a)) denoted by an asterisk (and simulating the location of the Ottawa Observatory) much exceeds the value $10^{-2} \mathrm{nT}$. Taking into account that the resolution of modern magnetometers is $10^{-2}$ nT (e.g. Hermanus, 2004), we may consider that value as the maximum allowable noise level at observatories at which precise instrument calibrations and recordings of small variations of the geomagnetic field with sensitive equipment are carried out. In a study of possible interferences produced by a power substation to the Kakioka Magnetic Observatory, Japan, Koike et al. (2004) conclude the magnetic disturbance to be less than $10^{-2} \mathrm{nT}$ at the observatory and say that it is negligible, which may be regarded as an additional indirect justification to regard $10^{-2} \mathrm{nT}$ as a noise limit. To achieve that level the distance should be about 20...30 km in Fig. 6(b).

\section{Measurements}

In order to verify the validity of the theoretical model discussed in Sections 2 and 3, measurements of the magnetic field were carried out in March 2006 for about a week at different distances from a nearly straight north-south dcelectrified railway in Calgary, Canada, already referred to in Fig. 4. Based on geological information, the ground structure in the area quite well satisfies the requirement of the theoretical model to be layered. The distances used in the recordings were about $0.6,1.0,1.5,2.0,2.6,3.3,3.7,4.7$, $5.5,8.0,12.0,21.9,27.0,50.0 \mathrm{~km}$. The measurement at 2.0 $\mathrm{km}$ was operating during the whole period and thus constitutes a reference. The magnetic signal due a train superimposed on the natural geomagnetic variation field is clearly detectable only until $5.5 \mathrm{~km}$ from the railway. Besides, the strength of the signal varied from case to case probably due to some differences in the amount of the current taken by the train. This was compensated in the analysis by scaling the data by using matching at the reference site $(2.0 \mathrm{~km})$. To get the largest numbers, we consider the absolute values of the magnetic disturbances due to the railway. Table 1 then shows the measured data.

Concluding from the recorded data and other information about train timetables, it is obvious that the magnetic disturbances shown in Table 1 were produced by one single train at a time. Therefore, in the theoretical calculations concerning a straight north-south railway, we consider one train and its feeding current system and assume that the points of observation are located at $x=0$ in the coordinate system described in the beginning of Section 2 and in Fig. 1, and the distance is equal to the $y$ coordinate. The values $3 \mathrm{~km}, 5 \mathrm{~m}$, 
Table 1. Absolute value of the magnetic disturbance caused by a nearly straight north-south dc railway as a function of the distance to the east. The data are based on measurements in Calgary, Canada, in March 2006.

\begin{tabular}{cc}
\hline Distance $[\mathrm{km}]$ & Magnetic Field $[\mathrm{nT}]$ \\
\hline 0.6 & 4.0 \\
1.0 & 2.1 \\
1.5 & 1.5 \\
2.0 & 0.9 \\
2.6 & 0.525 \\
3.3 & 0.45 \\
3.7 & 0.42 \\
4.7 & 0.3 \\
5.5 & 0.31 \\
\hline
\end{tabular}

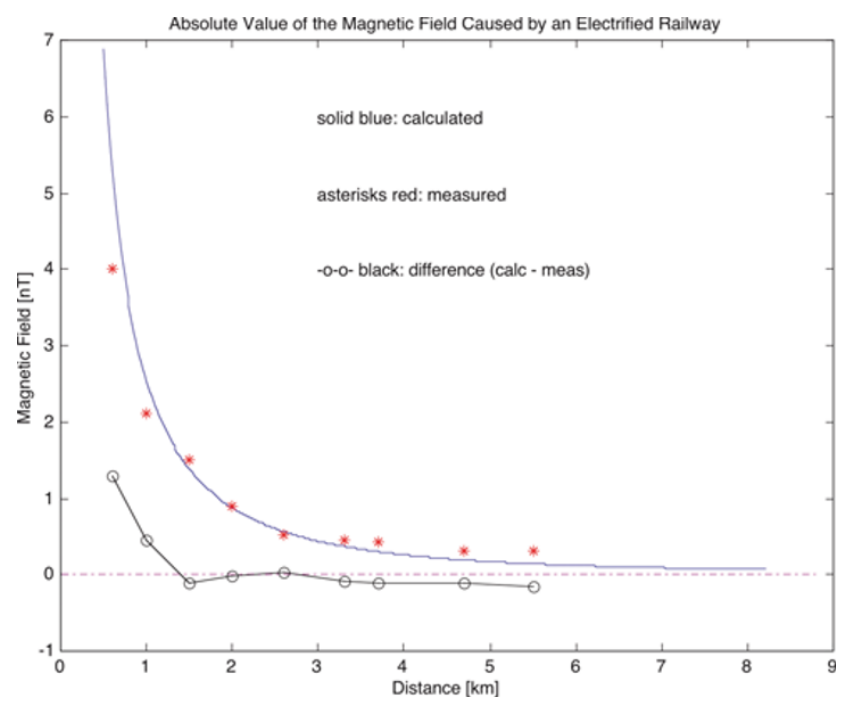

Fig. 7. Absolute value of the magnetic field created by the nearly straight north-south dc railway in Calgary, Canada. The red asterisks and the solid blue curve depict measured data and calculated results, respectively. The black circles show their difference. It is assumed that there is only one train powered by a substation located to the south at a distance of $3 \mathrm{~km}(=L)$, and the height of the feeding line and its current are $5 \mathrm{~m}(=h)$ and $1000 \mathrm{~A}\left(=J_{1}\right)$. The total leakage current is $20 \mathrm{~A}$ $\left(=J_{0}\right)$. The eastward line perpendicular to the railway along which the distance is measured is assumed to intersect the railway at the substation.

$1000 \mathrm{~A}$ and $20 \mathrm{~A}$ are used for the parameters $L, h, J_{1}$ and $J_{0}$, respectively.

Figure 7 shows a comparison between the measured and calculated absolute values of the magnetic field. The difference between calculations and measurements is also presented in the figure. Taking into account the inaccuracies in the measured data, including the determination of the distances based on the GPS and on some assumptions about the geometry possibly resulting in errors of roughly 0.1 $\mathrm{km}$ and the above-mentioned scaling, and the uncertainties in choosing the parameter values in the calculations, the agreement between the computations and the measurements can be regarded as excellent. Consequently, the recordings made in Calgary clearly demonstrate the validity of the theoretical model.

\section{Discussion}

Electric currents produced by man-made systems, for example electrified railways, create electric and magnetic fields that can seriously disturb geomagnetic observatory recordings and other electromagnetic studies even at distances of tens of kilometres. For observatories, at which precise instrument calibrations and recordings of small variations of the geomagnetic field by sensitive equipment obviously dictate the maximum allowable noise level to be about $10^{-2} \mathrm{nT}=10 \mathrm{pT}$ today, magnetic fields caused by dc railways are especially problematic. If the powering system of the trains would be ideal so that the return current (typically of the order of $1000 \mathrm{~A}$ ) would flow along the rails back to the substation the magnetic fields produced by the current in the feeding wire and the return current would tend to cancel each other making the disturbed area be limited to the vicinity of the railway. However, the rails always have electric connections to the soil due to intentional groundings for safety reasons or due to non-perfect insulation. Therefore a part (typically tens of amperes) of the return current leaks into the earth, which means that the cancellation gets weaker and the leakage currents in the earth create their own magnetic fields.

In this paper, adopting the model by Georgescu et al. (2002), we derive theoretical formulas for calculating the magnetic field at any point of observation due to a dc railway. The number of train-substation pairs, their locations and orientations, the lengths and heights of the feeding current wires and the feeding and leakage currents are arbitrary and just need to be specified by the user of the model. We have prepared the software in the MatLab, Octave, FORTRAN and IDL languages.

Based on calculations, the above-mentioned maximum allowable noise level of $10 \mathrm{pT}$ at an observatory seems to be a requirement difficult to be satisfied unless the distance between the observatory and dc railways is at least about $30 \mathrm{~km}$. The magnetic field created by a dc railway much depends on the magnitude of the leakage current, which may vary with insulation quality changes and with weather conditions. For different values of the leakage currents as well as of other partly unknown parameters related to dc trains, the conclusions drawn need not be quantitatively valid, but the computations can easily be re-performed with corrected input parameters by using the theoretical formulas presented in this paper.

Measurements of the magnetic field due to a dc train as a function of the distance from the railway in Calgary, Canada, are in a good agreement with results computed by applying the theoretical model, which validates the latter as depicted in Fig. 7. However, looking at the figure carefully, it is seen that the model tends to overestimate and underestimate the magnetic field at small and large distances, respectively. Near the railway, where the field changes rapidly with the distance, the discrepancies are probably affected by inaccuracies of the positioning of the measurement sites. Furthermore, the scaling of the data necessary to be made as explained in Section 4, may result in errors. Finally, the theoretical model was based on the assumption that the conductivity of the earth does not have lateral variations making the leakage current be distributed symmetrically in the 
ground. Lateral inhomogeneities cause that a bigger amount of the leakage current may flow to some directions obviously increasing the magnetic disturbance level there. This could be a subject of future studies. It may also provide an explanation to the differences in measured and calculated data in Fig. 7 although, as mentioned in Section 4, the ground structure is quite ideal in the area. Symmetric distribution of ground currents is certainly distorted if, for example, groundings of electric power grid substations or buried pipelines are present. This may be a subject of future investigations as well, which would also be important from the technological viewpoint as a current flow between a metal structure, e.g. a pipeline, and the soil can create problems associated with corrosion and unwanted disturbing voltages may occur in the equipment.

An additional issue that would need further considerations is the fact that the currents powering trains are not strictly dc and may include even quite high frequencies in their spectra. This means that induction effects in the earth may affect the paths of leakage currents and thus have some significance to the magnetic disturbance fields, too.

\section{Concluding Remarks}

Railways operating by dc currents create magnetic fields that can cause problems to geomagnetic observatories. These magnetic disturbances are mostly due to return currents leaking into the ground. In this paper, following a previous work by Georgescu et al. (2002), we present a theoretical model and derive formulas for calculating the magnetic field at any point of observation due to a dc railway occupied by one or several trains. The validity of the model was verified by measurements in the vicinity of a railway. Computer programs written in the MatLab, Octave, FORTRAN and IDL languages are readily applicable to any dc railway configuration. Numerical computations discussed in this paper indicate that magnetic fields larger than $10 \mathrm{pT}$ to be considered as the maximum allowable noise level at today's high precision observatories extend to distances of even tens of kilometres from a railway.

Acknowledgments. The authors wish to thank Mr. Lasse Häkkinen (Finnish Meteorological Institute) for his assistance in computer-technical matters. The two referees (Dr. Ikuko Fujii and Dr. Alan Thomson) of the manuscript are also gratefully acknowl- edged for many useful and constructive comments and suggestions that helped improve the paper.

\section{References}

Egbert, G. D., M. Eisel, O. S. Boyd, and H. F. Morrison, DC trains and Pc3s: Source effects in mid-latitude geomagnetic transfer functions, Geophys. Res. Lett., 27(1), 25-28, 2000. Correction in Geophys. Res. Lett., 27(10), 1565, 2000.

Fukushima, N., Generalized theorem for no ground magnetic effect of vertical currents connected with Pedersen currents in the uniformconductivity ionosphere, Report of Ionosphere and Space Research in Japan, 30(1/2), 35-40, 1976.

Georgescu, P., J.-L. Le Mouel, and M. Mandea, Electric and magnetic perturbations generated by d.c. electric railway, Geofizica, Bucuresti, Romania, 40, 69-82, 2002.

Hermanus Magnetic Observatory, Magnetic Results 2004: Hermanus, Hartebeesthoek and Tsumeb observatories, HMO Hermanus Magnetic Observatory, A facility of the National Research Foundation, South Africa, $40 \mathrm{pp}$.

Iliceto, V. and G. Santarato, On the interference of man-made EM fields in the magnetotelluric 'dead band', Geophysical Prospecting, 47, 707719, 1999.

Koike, K., T. Tokumoto, S. Nakajima, T. Owada, T. Ookawa, and H. Hasegawa, Geomagnetic and geoelectric field disturbance produced by a substation-Case study of Yasato Substation, Technical Report of the Kakioka Magnetic Observatory, 2(2), 51-67, 2004.

Kovalevskiy, I. V., N. V. Mikerina, V. V. Novysh, and O. P. Gorodnicheva, Distribution of the earth currents from an electrified railroad in the southern Urals, Geomagnetism and Aeronomy, I(1), 723-726, (translated by Scripta Technica, Inc. for the American Geophysical Union), 1961.

Lowes, F. J., Magnetic monitoring of dc electric railways, Phys. Technol., 18, 209-214, 1987.

Pádua, M. B., A. L. Padilha, and Í. Vitorello, Disturbances on magnetotelluric data due to DC electrified railway: A case study from southeastern Brazil, Earth Planets Space, 54(5), 591-596, 2002.

Tanbo, T., H. Sakai, and T. Nagao, A Study of Geoelectric Potential Change Caused by Rail Leak Current Observed at Ohtawa, Gifu, Japan, Electrical Engineering in Japan, 143(2), 10 pp., (translated from Denki Gakkai Ronbunshi, 122-A(5), May 2002, 446-453), 2003.

Tokumoto, T. and S. Tsunomura, Calculation of Magnetic Field Disturbance Produced by Electric Railway, translated from Memoirs of the Kakioka Magnetic Observatory, 20(2), 33-44, (pages 27-37 in the English translation), 1984.

Yanagihara, K., Magnetic Field Disturbance Produced by Electric Railway, Memoirs of the Kakioka Magnetic Observatory, suppl. 7, Geophys. Mag., 38(1), 17-35, 1977.

R. Pirjola (e-mail: risto.pirjola@nrcan.gc.ca; risto.pirjola@fmi.fi), L. Newitt, D. Boteler, L. Trichtchenko, P. Fernberg, L. McKee, D. Danskin, and $\mathrm{G}$. Jansen van Beek 